\title{
First-in-man endovascular preconditioning of the paraspinal collateral network by segmental artery coil embolization to prevent ischemic spinal cord injury
}

\author{
Christian D. Etz, MD, PhD, ${ }^{a}$ E. Sebastian Debus, MD, PhD, ${ }^{b}$ Friedrich-Wilhelm Mohr, MD, PhD, ${ }^{a}$ and \\ Tilo Kölbel, MD, $\mathrm{PhD}^{\mathrm{b}}$
}

\begin{abstract}
Objective: Spinal cord injury remains an invincible complication affecting up to 1 of 5 patients after thoracoabdominal aortic aneurysm repair. A staged surgical approach has been suggested to eliminate paraplegia by induction of arteriogenesis within the paraspinal collateral network; however, its clinical implementation was deferred because it required 2 major procedures.
\end{abstract}

\begin{abstract}
Methods: First-in-man experience with minimally invasive, selective segmental artery endovascular coil embolization before Crawford type II and III thoracoabdominal aortic aneurysm repair for arteriogenic preconditioning of the collateral network is reported. A 45-year-old man received bilateral elective minimally invasive, selective segmental artery endovascular coil embolization of 2 unilateral lumbar segmental arteries 4 weeks before open surgical repair. A 67-year-old woman who was planned for total endovascular treatment received minimally invasive, selective segmental artery endovascular coil embolization at the fourth lumbar level and coil embolization of the inferior mesenteric artery 8 weeks before endovascular repair.
\end{abstract}

Results: Minimally invasive, selective segmental artery endovascular coil embolization was technically successful and did not result in any neurologic impairment. Both patients were discharged after 72 hours of clinical surveillance. Patient 1 returned for definite open single-stage thoracoabdominal aortic aneurysm repair after 4 weeks and left the hospital without any neurologic impairment on day 35 postoperatively. Patient 2 returned for total endovascular repair 8 weeks after she underwent minimally invasive, selective segmental artery endovascular coil embolization; she recovered well and was discharged without spinal cord injury 8 days after extensive single-stage endovascular thoracoabdominal aortic aneurysm repair. Both patients are alive with no neurologic injury at 1-year follow-up.

Conclusions: Minimally invasive, selective segmental artery endovascular coil embolization for arteriogenic preconditioning of the paraspinal arterial collateral network is clinically feasible and may eventually eliminate ischemic spinal cord injury to enable safe open or endovascular repair of extensive thoracoabdominal aortic aneurysms and prevent paraplegia altogether in the near future. (J Thorac Cardiovasc Surg 2015;149:1074-9)

See related commentary on pages 1079-80.

Aortic disease, including thoracoabdominal aortic aneurysm (TAAA), is the 12th leading cause of overall death in the United States: Approximately 45,000 patients die annually of diseases of the aorta and its branches. ${ }^{1}$ The majority of patients with TAAA are septuagenarians and

From the Department of Cardiac Surgery, ${ }^{a}$ University of Leipzig, Heart Center Leipzig, Leipzig, Germany; and Department of Vascular Medicine, ${ }^{\mathrm{b}}$ University Heart Center, University of Hamburg, Hamburg, Germany.

This study was funded by an institutional research grant from the Leipzig Heart Center.

Received for publication April 29, 2014; revisions received Nov 26, 2014; accepted for publication Dec 6, 2014.

Address for reprints: Christian D. Etz, MD, PhD, Heart Center Leipzig, Struempellstrasse 39, 04289 Leipzig, Germany (E-mail: christian.etz@med. uni-leipzig.de).

$0022-5223 / \$ 36.00$

Copyright (c) 2015 by The American Association for Thoracic Surgery

http://dx.doi.org/10.1016/j.jtcvs.2014.12.025 octogenarians and cannot tolerate the daunting physiologic insult of open surgical repair. As a consequence, the majority of patients with TAAA are treated conservatively because of the prohibitive operative mortality and morbidity. TAAA surgery has achieved remarkable success over the past 50 years, particularly with the development of the endovascular techniques during the past decade, now offering durable operations with a low perioperative mortality and morbidity except for 1 imminent complication: ischemic spinal cord injury. Various perioperative neuroprotective strategies, that is, motor evoked potentials/somatosensory evoked potentials-guided segmental artery (SA) reimplantation, blood pressure management, continuous cerebrospinal fluid (CSF) drainage, ${ }^{2}$ and local spinal cord cooling, have been introduced to minimize spinal cord ischemia (SCI). These methods have achieved a remarkable decrease in the incidence of paraplegia and paraparesis, ${ }^{3,4}$ but they still constitute an incidence of no less than $10 \%$ to $20 \%$. The role of the Adamkiewicz artery as the single determining 


\section{Abbreviations and Acronyms \\ $\mathrm{CSF}=$ cerebrospinal fluid \\ CT = computed tomography \\ MISACE $=$ minimally invasive, selective segmental artery coil embolization \\ SA $=$ segmental artery \\ SCI $=$ spinal cord ischemia \\ TAAA $=$ thoracoabdominal aortic aneurysm}

SA for spinal cord blood supply recently has been questioned, and a new collateral network concept for spinal cord perfusion has been presented..$^{5-8}$ This concept includes the idea of a remodeling process driven by pressure differences across the collaterals, suggesting a staged approach to potentially reduce paraplegia by arteriogenic preconditioning, as recently demonstrated in an animal model. ${ }^{9,10}$ These experimental results have been clinically evaluated in a large retrospective analysis of TAAA repairs. ${ }^{11}$ Thereafter, the "staged approach" was introduced into the clinical practice of open and endovascular TAAA repair in selected centers requiring 2 procedures with open exposure or large-bore endovascular access and general anesthesia. Driven by the idea to trigger preconditioning of the collateral network endovascularly, selective SA coil embolization for staged repair was successfully developed experimentally. ${ }^{12}$ We describe the first-in-man clinical application of endovascular coil embolization as a first stage to precondition the arterial paraspinal collateral network before open and totally endovascular extensive TAAA repair.

\section{MATERIALS AND METHODS}

Two high-risk patients with large TAAAs, one planned for elective open endovascular repair and one planned for total endovascular repair, were offered minimally invasive, selective SA coil embolization (MISACE) because of expected sacrifice of an extensive number of patent SAs and an excessive risk of ischemic spinal cord injury.

Individual procedure planning was performed by an interdisciplinary team approach involving an endovascular and a cardiovascular surgeon based on computed tomography (CT) angiography and 3-dimensional reconstructions. Target SAs for coil deployment were identified considering the extent of the planned repair and individual SA anatomy.

The first stage of the treatment was performed under local anesthesia by embolization of the SAs of the thoracic or abdominal level. The procedure aims to occlude the vessels in their ostial segment to preserve the ability of the collateral network to compensate for these occlusions to a maximum extent. This procedure is done without spinal fluid drainage because the patient stays awake and can be continuously be monitored for neurologic function for a minimum of 48 hours after the procedure.

Although our laboratory data repeatedly suggested a time interval of 5 days to be sufficient to trigger the desired collateral network response (and achieve independence from direct segmental inflow), we decided to postpone definite repair further to allow for an additional safety margin in this first-in-man application. A time interval of at least 4 weeks in the adult patient was considered sufficient to allow for resilient preconditioning of the collateral network in the adult patient (based on clinical arteriogenesis data) to safely proceed to the definite open or endovascular TAAA repair performed under standard neuroprotective strategies.

Ethics approval is waived at our institution if all conventional and established treatment options carry a risk of death or devastating and substantially disabling complications (ie, permanent paraplegia), and new treatment options with limited clinical experience, using devices with $\mathrm{CE}$ mark or Food and Drug Administration approval (eg, coils) not specifically off label (ie, for the occlusion of intercostal arteries), are admissible as a last resort under the concept of individual compassionate use.

\section{Patient 1}

A 45-year-old asymptomatic man with a medical history of arterial hypertension and obesity was accidentally diagnosed with a Crawford type III TAAA with a maximum diameter of $65 \mathrm{~mm}$ extending from the descending thoracic to the infrarenal aorta (Figure 1, A) when CT imaging was obtained for chronic back pain; his configuration suggested a high risk for ischemic spinal cord injury (SCI) and consecutive permanent paraplegia with the aneurysm anatomy not suitable for a conventional, staged repair. His obesity was considered a risk factor for prolonged postoperative hospital course and interval mortality. With a considerable risk for sudden rupture and his indecisiveness about surgery because of the fear for paraplegia, the patient was offered MISACE for collateral preconditioning 4 weeks before elective open surgical repair.

Through a left common femoral artery access using a $6 \mathrm{~F}(30-\mathrm{cm}$ braided sheath; Flexor, Cook Medical, Bjaeverskov, Denmark) sheath in local anesthesia, the third and fourth lumbar SAs were catheterized with a $5 \mathrm{~F}$ 65- $\mathrm{cm} \mathrm{C1}$ catheter (Cook Medical), and a 2.7F Progreat microcatheter (Terumo Europe) and 7 microcoils $\left(0.018^{\prime \prime}\right.$ VortX-Diamond, Boston Scientific, Nanterre, France) were deployed in the proximal SA (Figure 1,B). No stable catheter position could be achieved in the left fourth SA because of severe tortuosity in the aortoiliac arteries.

One month later, conventional single-stage repair was performed, using adjunctive CSF drainage, extracorporeal circulation, and intraoperative neuromonitoring using motor-evoked potentials, with visceral artery reimplantation and bypass grafting to the right renal artery.

\section{Patient 2}

The second patient was a 66-year-old woman, with a medical history of arterial hypertension initially diagnosed during routine abdominal ultrasound examination, who had a rapidly growing Crawford type II TAAA with a maximum diameter of $57 \mathrm{~mm}$ and a rapid expansion of 5 $\mathrm{mm}$ within the past 6 months, extending from the descending to the infrarenal aorta (Figure 2, $A$ ). She was too frail for open repair, and totally endovascular treatment was planned. Because there was no option for conventional staging and the procedure was considered high risk for permanent paraplegia because the coverage of numerous SA was inevitable, she received elective bilateral MISACE of her fourth lumbar SAs and the inferior mesenteric artery 8 weeks before endovascular repair for collateral preconditioning: Through a right common femoral artery access and a $6 \mathrm{~F}$ sheath (30-cm braided, Flexor, Cook Medical), the fourth SAs were catheterized in local anesthesia with a $5 \mathrm{~F} 65-\mathrm{cm} \mathrm{C} 2$ catheter (Cook Medical), and a 2.7F Progreat microcatheter (Terumo Europe, Leuven, Belgium) and 5 microcoils ( $0.018^{\prime \prime}$ VortX-Diamond, Boston Scientific) were deployed in the proximal SA. The same procedure was conducted with the inferior mesenteric artery using 2 MReye macrocoils (Cook Medical) (Figure 2, B); no further open SAs were identified in the infrarenal segment.

Endovascular repair with a 4-branched stent-graft (T-branch, CE marked, Cook Medical) was performed under general anesthesia with adjunctive CSF drainage, occluding all remaining open SAs between the thoracic segment 7 and the infrarenal aorta (Figure 2,C).

\section{RESULTS}

MISACE was technically successful in both patients. Endovascular coil embolization did not result in any 

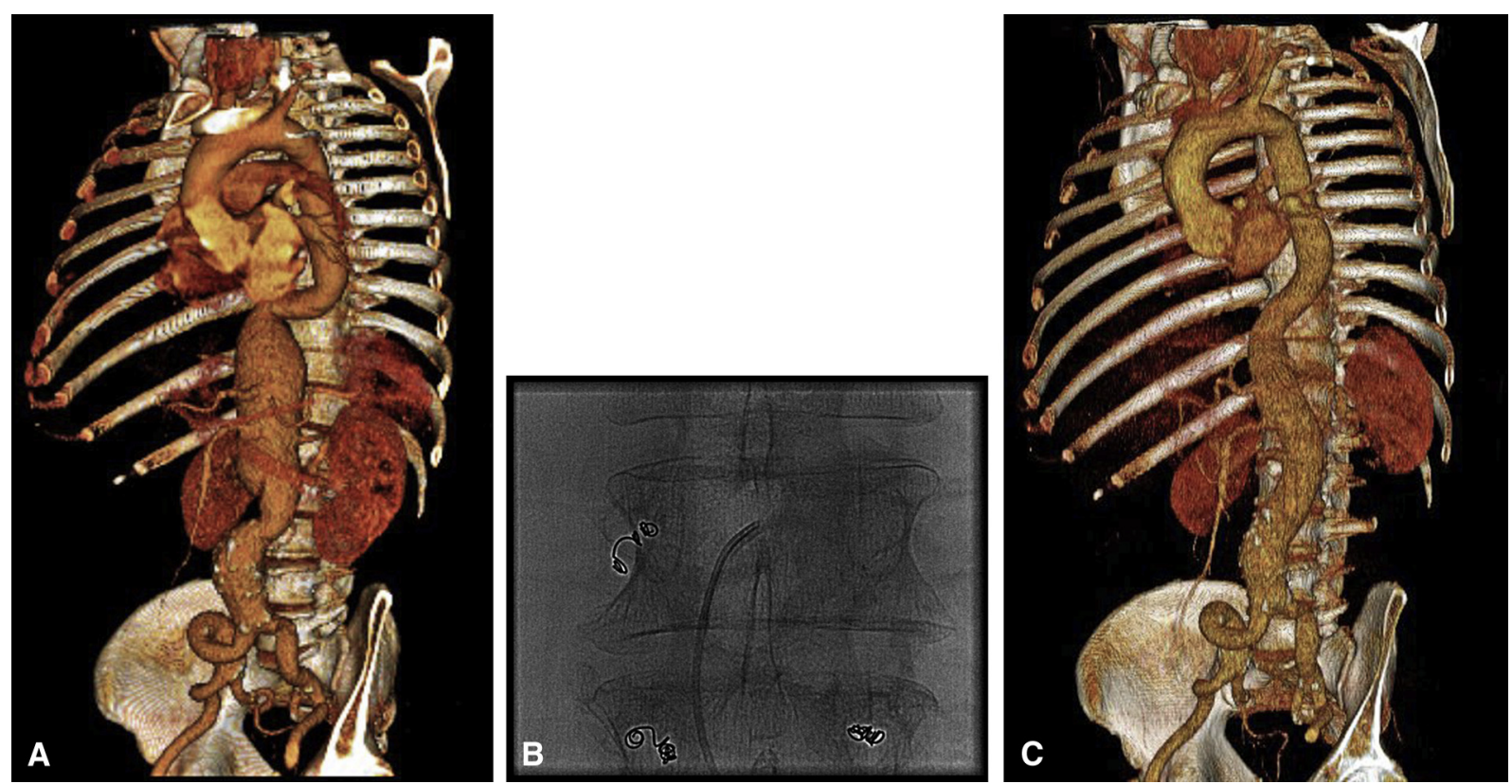

FIGURE 1. Patient 1 with a Crawford type III thoracoabdominal aortic aneurysm. A, Three-dimensional volume rendering of preoperative computed tomography angiography. B, Fluoroscopy demonstrating microcoils in 3 major segmental arteries. C, Three-dimensional volume rendering of the 5-month postoperative computed tomography angiography.

neurologic impairment, and both patients were discharged after 72 hours of clinical surveillance. Both patients were readmitted for definite aneurysm repair after an interval of 4 and 8 weeks, respectively.

The intraoperative course was uneventful in both patients: In patient 1 (open repair), aortic crossclamp time was significantly reduced because only a few pairs of patent, back-bleeding SAs remained after coil embolization, requiring occlusion by suture to preserve spinal cord perfusion pressure. On postoperative day 1, acute renal failure occurred after thrombotic occlusion of the right renal artery bypass graft and the patient was returned to the operating room; during the following hospital course, he also developed a proximal deep venous thrombosis and a wound infection requiring repeated debridements. After the patient's renal function had normalized, he left the intensive care unit on postoperative day 15 ; he had no neurologic impairment at any time and was discharged without on postoperative day 35 . On clinical follow-up at 5 months, renal function was restored, no neurologic impairment was present, and CT angiography showed an excellent result with unimpeded flow to all reimplanted visceral arteries (Figure 1, C).

In patient 2, totally endovascular TAA repair resulted in successful complete aneurysm exclusion. Postprocedural angiography revealed successful stent graft deployment with no endoleakage. During the postoperative course, the patient had no neurologic impairment at any time and was discharged on postoperative day 8 with no neurologic deficit. Intensive care unit stay was not necessary. On clinical follow-up at 4 months, no neurologic impairment was present and CT angiography showed an excellent result with unimpeded flow to all target arteries (Figure 2,D).

At 1-year follow-up, both patients were alive with no late neurologic impairment; postoperative CT angiography revealed freedom from type 2 endoleaking in both patients.

\section{DISCUSSION}

Ischemic SCI remains the most devastating complication after TAAA repair of any modality ${ }^{13}$ and is an individual disaster with a profound impact on long-term outcome and health care cost. ${ }^{14,15}$ Despite various modern neuroprotective strategies, ${ }^{2-4}$ there has been a definite and seemingly irreducible incidence of paraplegia after open repair of extensive TAAA, ${ }^{3,16-18}$ still affecting up to $25 \%$ of patients with Crawford I, II, and III aneurysms after any treatment modality (open repair or stent grafting).

The existence of a paraspinal collateral system involving an extensive axial arterial network located in the spinal canal, the paravertebral tissues, and particularly the paraspinous muscles has been proposed recently. ${ }^{8}$ The configuration of this arterial network includes inputs not only from the SAs (intercostal and lumbar) but also from the subclavian and the hypogastric arteries. The presence of this extensive network implies a considerable reserve to ensure spinal cord perfusion when some inputs are compromised.

This new concept ${ }^{5-8}$ has borne the idea of a remodeling process driven by local pressure gradients across the arterial collaterals that could lead a staged approach to potentially reduce SCI and permanent paraplegia by 

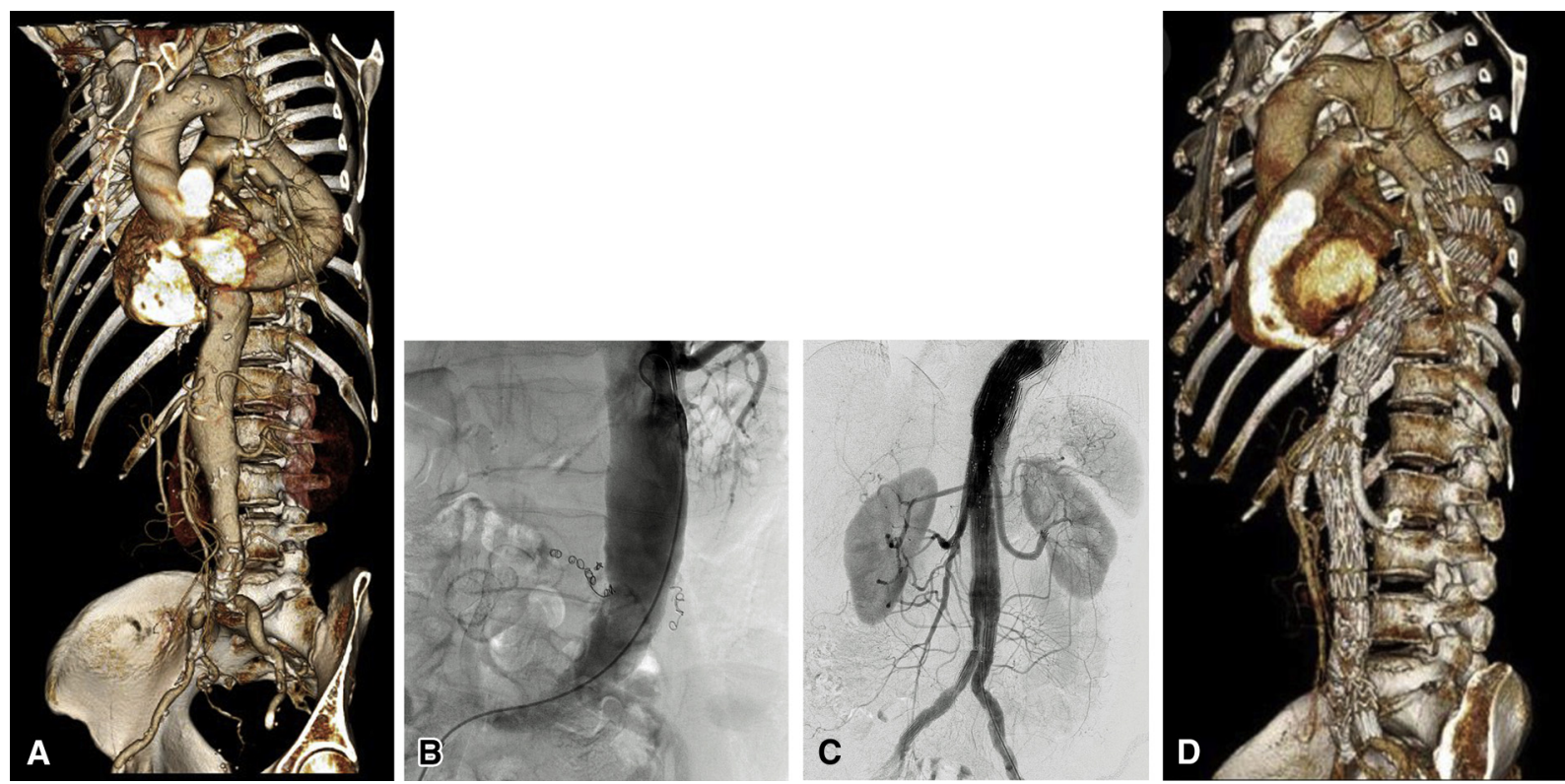

FIGURE 2. Patient 2 with a Crawford type II thoracoabdominal aortic aneurysm. A, Three-dimensional volume rendering of preoperative computed tomography angiography. B, Digital subtraction angiography demonstrating microcoils in 2 major segmental arteries and macrocoils in the inferior mesenteric artery. C, Completion angiography after branched endografting (white arrows point at coils in lumbar segmental arteries and inferior mesenteric artery). D, Three-dimensional volume rendering of postoperative computed tomography angiography.

arteriogenic preconditioning. ${ }^{9,10}$ Meanwhile, promising experimental results have been clinically evaluated ${ }^{11}:$ In 2010, a staged approach was suggested for the first time to significantly reduce paraplegia rate after extensive TAAA repair in a retrospective matched-pair analysis of a large clinical series identifying 35 patients with a Crawford II TAAA who had unintentionally undergone a 2-staged repair resulting in zero paraplegia. Particularly appealing about this staged approach is its applicability for TAAA repair of any modality: endovascular, open, and modern hybrid repair concepts. Although the staged approach has been successfully translated into clinical practice in select patients, the fact that it requires 2 procedures with open exposure or a large-bore endovascular access and general anesthesia remains dissuasive.

Various technical issues delayed translation of this concept into clinical practice (eg, the nondivisibility of most aneurysms rendering a staged approach demanding and at times impossible). It requires the secure occlusion of an appropriate number of selected SAs to induce a process of preconditioning within the arterial collateral network. Selective endovascular coil embolization, which can be performed under local anesthesia through a small transfemoral access in any catheter laboratory, has the potential to solve these issues and pave the way for a safe single-stage repair.

We describe the first-in-man clinical application of MISACE for collateral preconditioning before successful open and totally endovascular extensive TAA/A repair to minimize ischemic SCI. The selected patients had large and rapidly growing Crawford type II/III aortic aneurysms representing the classic dilemma: mandatory, urgent treatment exhibiting a particularly high risk of permanent SCI and technically highly demanding repair procedures not suitable for conventional staging:

(1) The elderly patient with a rapidly growing aneurysm, considered too frail to tolerate the daunting physiology of a staged open/hybrid repair with her only remaining option of single-stage endovascular treatment (ie, covering an extensive number of SAs in 1 session).

(2) The obese young man, considered high risk for acute rupture, requiring urgent, technically demanding, conventional repair (because of unknown long-term outcome after stent grafting) but facing high-risk surgery with extended aortic crossclamp time and significant interval mortality if a staged treatment was attempted.

MISACE offers a safe and simple innovation to potentially precondition the spinal cord's collateral network according to the modern collateral network concept before any modality of TAAA repair. MISACE provides all the benefits of a staged approach combined with the comfort of one definite single procedure that may be chosen to best suit the patient's needs technically and with regard to longevity of the repair.

MISACE for arteriogenic collateral network preconditioning before TAAA repair may be conducted during local anesthesia by direct coil embolization of SAs at the thoracic or abdominal level after analysis of the individual SA 
anatomy by $\mathrm{CT}$ angiography. The procedure aims to occlude the vessels proximally, in their ostial segment, to preserve the ability of the collateral network to compensate for these occlusions to a maximum extent. The procedure is performed without CSF drainage; however, the awake patient's neurologic function is meticulously monitored for a minimum of 48 hours after the procedure. After an appropriate time interval, the open or endovascular TAAA repair may be routinely performed using standard perioperative neuroprotective strategies.

Various complications have been described, and more may occur with the use of endovascular coils: (1) Risk of acute bleeding or rupture may be increased with oversizing or previous balloon occlusion of the SA (particularly with altered elasticity due to degenerative aneurysm disease or atherosclerotic disease); (2) free-floating loss of the coils in the aorta may cause undesired occlusion of visceral or peripheral end arteries; (3) a lack of complete thrombosis (eg, during oral anticoagulation or antiplatelet therapy) may occur and complicate its intended purpose of rapid and complete occlusion; (4) ischemic SCI and permanent paraplegia from iatrogenic occlusion of a large SA that may not undergo the desired physiologic collateralization may occur.

In this context, 2 equally threatening scenarios after coil deployment are conceivable: (1) acute SCI immediately after coil embolization and (2) delayed SCI, days, weeks, or months later, as has been described after both open and endovascular TAAA repair with extensive SA occlusion, that may be triggered by debris or microemboli from the aneurysm sack and incomplete occlusion of vital SAs in the presence of insufficient baseline collateralization through preexisting alternate pathways, yet insufficient preconditioning or steal phenomena (ie, blood flow directed toward watershed areas).

Options to deal with scenario 1 (acute immediate SCI after coil deployment) are limited and would include an endovascular rescue procedure to recover the coil and restore flow before irreversible SCI occurs or as a last resort to elevate systemic blood pressure to supranormal pressures to enforce saving blood flow to the spinal cord through sparse native collaterals. If delayed SCI should occur (scenario 2), bailout options are even more limited and would again include increasing systemic arterial pressure (avoiding drugs suspected to increase shunting) and to aggressively lower CSF pressure (if acute hemorrhage is excluded and steal phenomena are unlikely), both of which have been clinically described in such cases after TAAA repair with SA occlusion. If microemboli or debris is suspected (eg, generated or set free by the coil itself), and only if blood pressure elevation to supranormal blood pressure failed, thrombolysis could be considered as a last resort (with all its consequences) to rescue spinal cord function.
Delayed SCI seems to clinically (and according to our experimental experience) be most likely to occur during the first days after occlusion, and we therefore meticulously neuromonitored both patients on the IMC until discharge. Intensified experimental research is mandatory to learn more about these risks before application in low- or moderate-risk patients with SCI on a routine basis. Important synergistic side effects of MISACE preconditioning are reduced back-bleeding through SAs during open repair, reduced steal from the spinal cord and decreased aortic crossclamp times, and decreased type II endoleakage after complex endovascular repair after previously occluded culprit SAs bleeding into the excluded aneurysm sack.

\section{CONCLUSIONS}

With MISACE, minimally invasive staged preconditioning to significantly reduce iatrogenic SCI, the most devastating complication of any modality of TAAA repair, has become clinically available. Although MISACE to precondition the collateral network has clinically been attempted for the first time in man, the experimental data seem to be reasonably robust. MISACE may enable safer treatment of this life-threatening disease, in some cases with only 1 session in the catheter laboratory using widely available endovascular equipment, possibly influencing more patients in fear of paraplegia to undergo lifesaving aortic repair in the near future.

\section{Conflict of Interest Statements}

Sebastian Debus reports consulting fees from MedSkin and Molnlycke; lecture fees from Siemens, Medtronic, and Gore; and grant support from Cook Medical. Tilo Kölbel reports lecture fees and grant support from Cook Medical. All other authors have nothing to disclose with regard to commercial support.

\section{References}

1. Svensson LG, Kouchoukos NT, Miller DC, Bavaria JE, Coselli JS, Curi MA, et al. Expert consensus document on the treatment of descending thoracic aortic disease using endovascular stent-grafts. Ann Thorac Surg. 2008;85:S1-41.

2. Acher CW, Wynn MM, Archibald J. Naloxone and spinal fluid drainage as adjuncts in the surgical treatment of thoracoabdominal and thoracic aneurysms. Surgery. 1990;108:755-62.

3. Acher CW, Wynn MM, Mell MW, Tefera G, Hoch JR. A quantitative assessment of the impact of intercostal artery reimplantation on paralysis risk in thoracoabdominal aortic aneurysm repair. Ann Surg. 2008;248:529-40.

4. Acher C. It is not just assisted circulation, hypothermic arrest, or clamp and sew. J Thorac Cardiovasc Surg. 2010;140:S136-46.

5. Etz CD, Kari FA, Mueller CS, Brenner RM, Lin HM, Griepp RB. The collateral network concept: remodeling of the arterial collateral network after experimental segmental artery sacrifice. J Thorac Cardiovasc Surg. 2011;141:1029-36.

6. Etz CD, Kari FA, Mueller CS, Silovitz D, Brenner RM, Lin HM, et al. The collateral network concept: a reassessment of the anatomy of spinal cord perfusion. J Thorac Cardiovasc Surg. 2011;141:1020-8.

7. Etz CD, Zoli S, Bischoff MS, Bodian C, Di Luozzo G, Griepp RB. Measuring the collateral network pressure to minimize paraplegia risk in thoracoabdominal aneurysm resection. J Thorac Cardiovasc Surg. 2010;140:S125-30; discussion S142-6. 
8. Griepp RB, Griepp EB. Spinal cord perfusion and protection during descending thoracic and thoracoabdominal aortic surgery: the collateral network concept. Ann Thorac Surg. 2007;83:S865-9; discussion S890-2.

9. Bischoff MS, Scheumann J, Brenner RM, Ladage D, Bodian CA, Kleinman G, et al. Staged approach prevents spinal cord injury in hybrid surgicalendovascular thoracoabdominal aortic aneurysm repair: an experimental model. Ann Thorac Surg. 2011;92:138-46.

10. Zoli S, Etz CD, Roder F, Brenner RM, Bodian CA, Kleinman G, et al. Experimental two-stage simulated repair of extensive thoracoabdominal aneurysms reduces paraplegia risk. Ann Thorac Surg. 2010;90:722-9.

11. Etz CD, Zoli S, Mueller CS, Bodian CA, Di Luozzo G, Lazala R, et al. Staged repair significantly reduces paraplegia rate after extensive thoracoabdominal aortic aneurysm repair. J Thorac Cardiovasc Surg. 2010;139:1464-72.

12. Geisbusch S, Stefanovic A, Koruth JS, Lin HM, Morgello S, Weisz DJ, et al. Endovascular coil embolization of segmental arteries prevents paraplegia after subsequent thoracoabdominal aneurysm repair: an experimental model. J Thorac Cardiovasc Surg. 2014;147:220-6.

13. Coselli JS, LeMaire SA, Conklin LD, Koksoy C, Schmittling ZC. Morbidity and mortality after extent ii thoracoabdominal aortic aneurysm repair. Ann Thorac Surg. 2002;73:1107-16.
14. Bicknell CD, Riga CV, Wolfe JH. Prevention of paraplegia during thoracoabdominal aortic aneurysm repair. Eur J Vasc Endovasc Surg. 2009;37: 654-60.

15. Huynh TT, Miller CC 3rd, Estrera AL, Sheinbaum R, Allen SJ, Safi HJ Determinants of hospital length of stay after thoracoabdominal aortic aneurysm repair. J Vasc Surg. 2002;35:648-53.

16. Williams GM, Roseborough GS, Webb TH, Perler BA, Krosnick T. Preoperative selective intercostal angiography in patients undergoing thoracoabdominal aneurysm repair. J Vasc Surg. 2004;39:314-21.

17. Safi HJ, Miller CC 3rd, Carr C, Iliopoulos DC, Dorsay DA, Baldwin JC Importance of intercostal artery reattachment during thoracoabdominal aortic aneurysm repair. J Vasc Surg. 1998;27:58-68.

18. Cambria RP, Davison JK, Carter C, Brewster DC, Chang Y, Clark KA, et al. Epidural cooling for spinal cord protection during thoracoabdominal aneurysm repair: a five-year experience. J Vasc Surg. 2000;31:1093-102.

Key Words: spinal cord injury, staged TAA/A-repair, endovascular segmental coil—embolization

\title{
EDITORIAL COMMENTARY
}

\section{Setting the stage: Thoracoabdominal aortic aneurysm repair in 2 acts}

\author{
Grayson H. Wheatley III, MD
}

See related article on pages 1074-9

Ischemic spinal cord injury associated with thoracoabdominal aortic aneurysm (TAAA) repair remains a serious concern in patients treated with either open surgical or endovascular techniques. The advent of the collateral network concept by Griepp and Griepp in $2007^{1}$ significantly improved our understanding of the intricate anatomic and physiologic processes affecting spinal cord ischemia associated with aortic repair. Furthermore, the collateral network concept, for the first time, opened the theoretical possibility of staging aortic repair in the hopes

\footnotetext{
From the Division of Cardiovascular Surgery, Temple University School of Medicine, Philadelphia, $\mathrm{Pa}$.

Disclosures: Author has nothing to disclose with regard to commercial support.

Received for publication Dec 27, 2014; accepted for publication Dec 30, 2014; available ahead of print Feb 4, 2015.

Address for reprints: Grayson H. Wheatley III, MD, Temple University School of Medicine, 3401 N Broad St, 3rd Fl, Zone C, Ste 301, Philadelphia, PA 19140 (E-mail: grayson.wheatley@tuhs.temple.edu).

J Thorac Cardiovasc Surg 2015;149:1079-80

$0022-5223 / \$ 36.00$

Copyright (c) 2015 by The American Association for Thoracic Surgery

http://dx.doi.org/10.1016/j.jtcvs.2014.12.071
}

of recruiting collateral channels for spinal cord perfusion to compensate for loss of valuable intercostal arteries. ${ }^{2}$ However, the practicalities of staging aortic repair in association with recruiting the collateral network have, until now, been difficult to implement clinically. As has been said before, all the world is a stage and aortic surgery for TAAA has previously been a single-act play (ie, definitive aortic repair), whereas now there can be 2 acts with an intermission.

In this issue of the Journal of Thoracic and Cardiovascular Surgery, Etz and colleagues ${ }^{3}$ report the first-in-man endovascular ischemic preconditioning treatment to help prevent ischemic spinal cord injury. The technique of minimally invasive selective segmental artery coil-embolization (MISACE) described by the authors involves triggering recruitment of the spinal cord arterial collateral networks by first coil-embolizing important lumbar arteries that had been identified by preoperative computed tomography, followed by an interval of 4 weeks before definitive aortic repair. As noted in the title, the 2 patients treated as part of this study are the first reported successful cases of this staged approach to aortic repair.

There are several important breakthroughs relating to managing and preventing spinal cord injury that have been simultaneously brought together with the MISACE technique. The first is that optimal perioperative management of spinal cord function is not enough to prevent spinal 